\title{
La formación docente en Didáctica de las Ciencias Sociales a través de los proyectos socioeducativos en contextos de exclusión social
}

\section{Teacher training in Social Sciences Didactics through social and educations projects in exclusion contexts}

\section{María Dolores Jiménez Martínez Manuel José López Martínez Concepción Moreno Baró}

Universidad de Almería

\begin{abstract}
Resumen: Presentamos una experiencia formativa innovadora con los estudiantes de la titulación de Maestro/a de Educación Primaria de la Facultad de Ciencias de la Educación de la Universidad de Almería. Se ha realizado en el marco de un Programa Socioeducativo en colegios públicos de un barrio marginal de la ciudad de Almería mediante la metodología de Aprendizaje-Servicio (ApS). Desde la Didáctica de las Ciencias Sociales queremos contribuir a una educación democrática mediante la participación ciudadana. Mostramos algunas valoraciones de nuestros estudiantes en este proceso de investigación-acción. El balance positivo de esta evaluación inicial nos motiva para seguir implicados en el desarrollo de proyectos como éste donde se vincula universidad, escuela y comunidad.
\end{abstract}

Palabras Clave: formación inicial, evaluación, innovación docente, competencia social y ciudadana, aprendizaje-servicio.

Abstract: This paper speaks about an innovative training experience with Pre-Service Primary Teacher at the University of Almeria, Faculty of Education. Carried out in the framework of a social and educational project in public schools in a slum in the city of Almeria using service learning methodology. From the teaching of the social sciences our aim seeks to contribute to a democratic education through citizen participation. We show some evaluations carried out by our students in the process of action research. The positive results of this initial assessment motivates us to keep involved in the development of projects like this, where University, school and community are linked.

Key words: pre-service primary teacher, assessment, teaching innovation, social and civic competence, service-learning.

(Fecha de recepción: abril, 2014, y de aceptación: enero, 2015)

DOI: 10.7203/DCES.29.3534

\footnotetext{
* Este trabajo forma parte del "Proyecto socioeducativo por la recuperación de espacios públicos. El Ingenio", BOJA 14 de enero de 2009. A su vez ha sido desarrollado desde el Proyecto de Trabajo del Grupo Docente de Innovación "Aprendizaje Servicio: vinculando universidad, escuela y comunidad" de la Universidad de Almería avalado y financiado en la Convocatoria Bienal de Grupos Docentes para el Diseño y Transferencia Práctica de Innovaciones Docentes durante los cursos 2011-12/2012-13. En el momento de presentar este artículo salen a la luz datos sobre el aumento de exclusión social en España. La Fundación FOESSA, impulsada por Cáritas, presentó el 27 de marzo el estudio "Análisis y Perspectivas 2014". El trabajo revela que 11,7 millones de personas están afectadas en España por distintos procesos de exclusión social, un 60\% más que en 2007 (El País, 28 de marzo de 2014).
} 


\section{Introducción}

En este artículo presentamos las líneas fundamentales de una acción docente innovadora que venimos realizando en la titulación de Maestro/a de Educación Primaria de la Universidad de Almería así como los primeros avances de la evaluación inicial de la misma. Esta acción forma parte del Programa Socioeducativo de carácter comunitario para la recuperación de El Ingenio realizado en dos colegios públicos de la barriada marginal de El Puche en la ciudad de Almería. A su vez y de forma paralela este trabajo se desarrolla dentro del Grupo Docente de Innovación de la Universidad de Almería denominado Aprendizaje Servicio: vinculando universidad, escuela y comunidad donde trabajamos de forma colaborativa e interdisciplinar entre el profesorado de Didáctica y Organización Escolar y el de Didáctica de las Ciencias Sociales.

En el proceso investigador han participado de forma voluntaria estudiantes de $3^{\circ}$ de Magisterio de Educación Primaria (plan de 1999) y posteriormente de $2^{\circ}$ y $3^{\circ}$ de Grado Maestro/a en Educación Primaria (plan de 2011) y ha tenido continuidad hasta el curso 20122013. Del cómputo total del alumnado matriculado en las materias de Didáctica de las Ciencias Sociales en ambos planes de estudio (225 estudiantes), la muestra de estudiantes que se ha vinculado, participado y comprometido en la acción docente e investigadora realizada en los dos colegios del barrio marginal de El Puche, CEIP “Josefina Baró” y CEIP "El Puche", ha sido del 22\%, 50 en total a lo largo de los tres cursos académicos, 35 mujeres y 15 hombres. A priori, puede resultar una muestra muy reducida pero hay que considerar en primer lugar su carácter voluntario y al mismo tiempo el hecho de realizarse en contextos educativos complejos y de exclusión que crearon en un principio dudas y reservas en el alumnado universitario. Sin embargo, esta circunstancia ha sido un elemento de fortaleza para realizar la investigación-acción de acuerdo con la metodología etnográfica.

La justificación y relevancia de este Proyecto de Trabajo de Grupo Docente se sustenta en varias razones que podemos resumir en palabras de Teresa García (2011) de la siguiente manera:

“(...), la participación del alumnado en el Programa Socioeducativo para la recuperación de El Ingenio facilitará iniciar un nuevo modelo formativo, es decir, cambiar la propia concepción y práctica de formación del alumnado universitario, cuya formación se fundamentará en el espacio de intersección entre universidad, escuela y comunidad. Es decir, que la Universidad no sólo sea un lugar de transmisión de conocimiento, sino de reconstrucción del mismo a partir de una realidad concreta; el alumnado no sólo se formaría para una práctica profesional democrática, sino en una práctica profesional democrática, y nos ofrece la oportunidad de un modelo de formación permanente para nosotras mismas, caracteriza- 
do por el trabajo cooperativo e interdisciplinar con una metodología de investigación-acción".

Nuestra hipótesis de trabajo pretende demostrar que las competencias profesionales del o la docente se aprenden con mayor solidez reflexionando sobre la experiencia diaria en un espacio escolar de intersección entre Universidad, Escuela y Comunidad. Esta hipótesis se sustenta en tres objetivos específicos de este proyecto. A saber, la formación democrática participativa de nuestro alumnado, la integración formativa de los conocimientos teóricos y prácticos de la Didáctica de las Ciencias Sociales para su futura acción docente y el uso de la experiencia docente y su participación global en el proyecto para dar sentido a la labor del maestro o la maestra como agentes de transformación social. La potencialidad transformadora del análisis de la realidad que ofrecen las Ciencias Sociales es nuestro principal bastión para formar a docentes capaces de enseñar a construir otros futuros posibles más que necesarios ante el desaliento imperante en contextos de exclusión social.

Creemos que todavía persiste entre el alumnado en formación y en el mismo profesorado de Primaria en ejercicio un cierto rechazo hacia la formación recibida por carecer ésta de un sentido práctico. En esta línea, hemos pretendido generar un conocimiento amplio de los procesos de aprendizaje de la práctica educativa que experimenta y vive el alumnado universitario. Se trata de un conocimiento que nos ayuda como docentes-formadores a adaptar el proceso de enseñanza y a reorientar nuestra metodología desde la investigaciónacción. Todo ello dirigido a facilitar la adquisición de competencias profesionales por parte del o la futura docente de Primaria en los diferentes contextos educativos.

En el proceso de investigación-acción en que se funda este proyecto hemos podido obtener un conocimiento más preciso de las vivencias, experiencias y reflexiones de este alumnado que ha recibido una formación en Didáctica de las Ciencias Sociales. A lo largo de todo este tiempo hemos desarrollado una investigación cualitativa utilizando para ello diversas metodologías y técnicas etnográficas. Hemos simultaneado diversos instrumentos de recogida de datos como la observación de la realidad, la observación simple y participante, entrevistas formales e informales, grupos de discusión, análisis de diarios, de memorias y de diseños curriculares, cuestionarios abiertos y cerrados..., con el fin de verificar la consecución de las competencias profesionalizadoras.

Nuestra acción evaluadora se ha sustentado en tres aspectos relevantes. En primer lugar, vimos oportuno que el o la futura docente tomara conciencia de que la finalidad de la enseñanza de las Ciencias Sociales hoy, al transformarse en conocimiento escolar, ha de emplearse para solucionar esferas de la vida individual y social que se encuentran en una situación problemática. En segundo lugar, trasladamos la idea 
de que la enseñanza y aprendizaje de las Ciencias Sociales en Primaria ha de contribuir a la consolidación de una educación para una ciudadanía crítica y reflexiva en el contexto de una sociedad democrática, multicultural, capitalista y globalizada. Y, en tercer lugar, con nuestra acción formadora y evaluadora, vimos oportuno resaltar que el o la futura docente asumiera la responsabilidad de convertirse en un agente de iniciación en el cambio y en la transformación social.

El propósito ha sido incidir en el cambio del modelo formativo conectando universidad, escuela y comunidad, a través de la metodología del Aprendizaje-Servicio (ApS) para el desarrollo de la competencia social y ciudadana. Hemos pretendido generar un cambio en los procesos educativos que permita construir y desarrollar la democracia para una ciudadanía crítica, activa y participativa, vinculada a los problemas reales de la ciudadanía.

Como docentes de formación inicial del profesorado somos conscientes de la necesidad de construir propuestas formativas en el ámbito de la participación ciudadana en todos los niveles educativos, en este caso el universitario. Se trata de una propuesta de formación con carácter integrador y recíproco donde el eje principal ha sido formar al alumnado y formarnos como docentes universitarios para que vayan-vayamos construyendo una educación democrática desde la participación.

\section{Nueva dimensión en la educación para la ciudadanía en un contexto incierto}

La crisis actual está haciendo visible las fracturas y los problemas de las democracias actuales. Josep Ramoneda en su artículo de opinión "La democracia en peligro" (El País, 16 de enero de 2012) hizo un diagnóstico, creemos muy certero, de la situación de la democracia en Europa que ha entrado en franca pérdida de calidad. Su empequeñecimiento lo define con las siguientes características: la negación de la alternativa -la crisis ha llevado el principio "no hay alternativa" al paroxismo-; políticas del miedo -el discurso de la culpa colectiva-; satanización del conflicto -distintos sectores ideológicos sobre todo conservadores arremetieron contra los indignados por el atrevimiento de señalar la desnudez de la democracia e interrogarse por posibles alternativas-; cultura de casta -el complejo político-económico-mediático que aparece cada vez más alejado de la ciudadanía-; y las rupturas de las condiciones básicas de la igualdad -el crecimiento exponencial de la desigualdad hace que se pierda la condición propia de la sociedad democrática-. La fractura entre integrados y marginados es, en su opinión, una herida letal en el sistema democrático.

Ante esta "democracia en peligro", la educación ciudadana hoy más que nunca no puede quedarse en un discurso vacío con buenas intenciones; por el contrario tiene que dotarse de mecanismos de acción y sobre todo de 
participación. Es cierto, como señala Francisco García (2009), que la idea de la participación ciudadana es un concepto cada vez más presente en nuestra sociedad como pudimos observar en el movimiento de los indignados, $15-\mathrm{M}$, que trató de reinventar con orgullo la democracia con fórmulas más frescas y participativas ante esta crisis que además de económica y social es también ética y educativa (Carbonell, 2011). Aunque "satanizado" por ciertos sectores, no hay que minimizar el alcance y la singularidad del movimiento en el cambio de las prácticas ciudadanas.

La concepción tradicional de ciudadanía se ha visto modificada por los cambios económicos, políticos y sociales que se están produciendo en el mundo. El ejercicio del poder, la representación política, empiezan a adquirir nuevos significados, no sin conflictos, que demandan modificar el ideal democrático y exigen el ejercicio de una nueva ciudadanía. Estamos definiendo una ciudadanía global frente a la ciudadanía del Estado-Nación arraigada en la sociedad y en la tradición escolar. Una ciudadanía democrática auténtica, recuperando como elementos básicos la participación activa y el compromiso social, para poder superar la atonía de un ejercicio representativo que se ha mostrado sin recursos para afrontar los problemas actuales.

Se ha confirmado que los jóvenes españoles de hoy son los que tienen una mayor desafección política en relación con otros países de nuestro entorno. Son herederos de unas vivencias de la
Dictadura que ha dejado secuelas que desprecian lo político. Los discursos políticos son ajenos a ellos y no incitan a su participación. Tampoco disponen de recursos para la participación e implicación en el ámbito escolar. Actualmente, en el escenario incierto en el que las democracias occidentales se están desenvolviendo, el modelo de ciudadanía democrática y participativa que se pretendía conseguir mediante el sistema formal de la enseñanza obligatoria está siendo desprestigiado por parte de los discursos neoliberales del modelo económico capitalista. Como consecuencia de la globalización de este sistema, éste ha comenzado a ensalzar un discurso sustentado en una serie de valores fundamentados en el individualismo, desacreditando los currículos fomentados en los centros escolares públicos que conectan con aquellos otros que favorecen la convivencia democrática y la participación colectiva desde una reflexión crítica. Manuel Cruz (2012) describe las características de ese individualismo en los siguientes términos:

“(...) un individualismo en el que el repliegue de los sujetos sobre sí mismos se sustancia, bien en una banalidad sin ambición transformadora alguna, bien en una ansiedad provocada por la impotencia para incidir a tal escala en el desarrollo social y político de la realidad en la que viven."

En definitiva, la actual concepción y práctica de la ciudadanía se caracteriza 
por la pasividad, la despreocupación y asignación de responsabilidades colectivas del Estado por lo que los ciudadanos son meros espectadores de la acción política (Martínez, 2010).

Voces autorizadas (Morin, 2001; Tedesco, 2000) vienen defendiendo desde hace años una perspectiva global de la educación desde un enfoque complejo e integrador. En el contexto de la globalización con sociedades complejas y plurales, la educación para la ciudadanía no debe generar sentimientos patrióticos sino preparar para afrontar los problemas sociales que surgen en estas nuevas sociedades multiculturales.

La educación para la ciudadanía tiene que tener un componente de responsabilidad cívica; en este sentido la Comisión Europea, (Education for Democratic Citizenship in the European Union) plantea como objetivo la formación de una ciudadanía autónoma, crítica, participativa y responsable en una sociedad que respeta los principios de la democracia y los derechos humanos. En EE.UU. se concibe un modelo más participativo donde el alumnado participa activamente en experiencias de servicio integradas en el currículum. Efectivamente, los programas de "aprendizaje-servicio" (ApS) vinculan el servicio social y el aprendizaje en una sola acción educativa con la finalidad de ejercer la ciudadanía y promover el compromiso cívico a través de la acción. Parte de lo que en EE.UU. en los años 60 y 70 se entendía como community participation education, derivó a par- tir de los años 90 en service learning (Boyle-Baise y Grant, 2004).

La educación para la ciudadanía requiere, pues, "una dimensión más vinculada a la acción, una educación para la participación ciudadana, entendida en sentido amplio y en distintas escalas (en el propio barrio, en la ciudad, en el estado, en el Mundo...)" (García y De Alba, 2007). La ciudadanía en el mundo actual debe hacer interaccionar lo global y lo local, esto es, en un contexto local, el barrio por ejemplo, generar un espacio de participación desde donde reflexionar y trabajar conjuntamente lo local y lo global.

Por tanto, y ante los problemas sociales de nuestro mundo, la educación para la participación ciudadana debe integrar el compromiso, la corresponsabilidad y el sentido crítico ante las desigualdades y las injusticias sociales, así como el compromiso de lucha por un mundo más justo. Hablamos, pues, de una educación para la participación ciudadana que vincule los conocimientos con la intervención social.

\section{El Programa socioeducativo en un contexto de exclusión}

Los orígenes de este proyecto de recuperación de espacios públicos se remontan al año 2004 cuando la Consejería de Obras Públicas y Vivienda de la Junta de Andalucía encomienda a la Empresa Pública de Suelo de Andalucía (EPSA), la realización de un Proyecto de regeneración urbana para modificar la situación de este barrio. Se 
recogieron propuestas y acciones para una rehabilitación global además de su integración social y urbana en la ciudad de Almería. EPSA crea ese mismo año una Oficina de Gestión en El Puche para trabajar directamente con los vecinos, asociaciones, ONGs, Ayuntamiento de Almería y diversas organizaciones para definir entre todos cuáles tendrían que ser las diferentes actuaciones en el ámbito social, urbano y económico para regenerar y mejorar las condiciones de vida de sus habitantes. Se puso en marcha así el Programa de Actuación para la Rehabilitación Integral de El Puche.

Uno de los objetivos principales de este Programa era el de eliminar las principales barreras físicas, sociales y económicas detectadas mediante la implicación activa de vecinos y vecinas del barrio basándose para ello en el convencimiento de que para propiciar un proyecto sostenible en el tiempo era imprescindible construir espacios participativos de la ciudadanía para la toma de decisiones.

El barrio de El Puche se creó para paliar los efectos ocasionados por las lluvias torrenciales que cayeron en la capital almeriense durante los primeros días de enero de 1970 (Sánchez, S., García, T. 2010). Tras las lluvias se contabilizaron un total de 453 viviendas y cuevas derruidas, declarándose en ruina 2.118 casas y chabolas. Como consecuencia de ello, unas mil personas de los barrios más humildes de la capital: Barrio Alto, Cuevas de El Puche, Hoyo de las tres Marías, Chamberí, Cuevas de San Joaquín, San Cristóbal, etc., necesitaron ser alojadas en albergues provisionales.

El Consejo de Ministros aprobó el 23 de febrero de 1970 la construcción de 1.100 viviendas para las personas afectadas. Su emplazamiento se ubica en la finca "Cortijo Puche". Las obras comenzaron en mayo de 1971, con 996 casas aprobadas, de las cuales 496 serían de una sola planta, con $55,28 \mathrm{~m}^{2}$ útiles, localizándose en el denominado como Puche Centro o Puche Viejo y 500 viviendas de cuatro plantas, llamado Puche Norte, últimas viviendas en entregar. En 1975 la empresa encargada de construir las casas abandona su ejecución, quedando pendientes por terminar otras 500 casas. Tras una subasta se adjudicó la construcción de 427 viviendas tipo dúplex de diferentes tipologías que se terminaron en 1980 y a cuya ubicación se llamó Puche Sur o "Huevos fritos". El realojo se hizo sin planificación urbanística previa, con una absoluta carencia de servicios y desconectada del resto de la ciudad.

En un artículo de Arjona y Checa (2005), titulado "Factores que determinan el proceso de exclusión de los barrios periféricos: el caso de El Puche (Almería)", estos investigadores de la Universidad de Almería sostenían lo siguiente:

"Después de analizar los factores determinantes que influyen en el proceso de exclusión de este barrio, percibimos como fundamental la heterogeneidad interna tanto en la población, como en la configuración física, social, económica y residencial 
de este. Esta complejidad desemboca en distintos procesos de exclusión y demuestra una asimetría entre quienes gozan de una relativa estabilidad y quienes se encuentran inmersos en una total vulnerabilidad. Por tanto, El Puche puede estudiarse desde diferentes estadios y podemos afirmar que ninguno de los sub-barrios que lo componen estaría en la zona de integración y apenas existen individuos que tengan un empleo estable y bien remunerado, redes sociales, etc."

En estos últimos 40 años, El Puche, con una población de más de 7.000 habitantes, con gran presencia de inmigración (70\%), la mayoría marroquíes, el $15 \%$ de población gitana y el $15 \%$ no gitanos, ha experimentado una degradación progresiva. Hoy en día existe un conflicto en la convivencia debido a que la comunidad gitana se ha visto desplazada ante la llegada masiva de la población inmigrante marroquí asentada en el barrio con miras de permanencia. Otra problemática a destacar se concentra en las actitudes y comportamientos incívicos de sus habitantes en los espacios públicos comunitarios.

El Proyecto Socioeducativo de carácter Comunitario para la Recuperación de "El Ingenio-El Puche" (Sánchez y García, 2010) vincula la educación formal y no formal para la transformación del barrio. Pretende ser una propuesta educativa y al mismo tiempo dar servicio a la comunidad. Desde las pedagogías activas, el Aprendizaje-Servicio (ApS) como metodología de trabajo del pro- yecto significa una manera de entender la ciudadanía: basada en la participación activa y la contribución a la mejora de la calidad de vida de la sociedad; una manera de entender el aprendizaje basada en la responsabilidad social, la exploración, la acción y la reflexión y una forma de entender la educación en valores basada en la vivencia, la experiencia y la construcción de hábitos. En definitiva, una propuesta pedagógica que quiere responder a necesidades sociales al tiempo que se aprende y se vive una experiencia significativa colaborando con otras instituciones sociales con las que se establecen vínculos asociativos (Batllé, 2010; Puig, 2009).

La experiencia formativa que venimos desarrollando se realiza en una comunidad donde la participación ciudadana se ha convertido en el motor de la transformación del barrio de El Puche en la ciudad de Almería. La especificidad del mismo, catalogado en su momento por la Administración Pública como Zona con Necesidades de Transformación Social (ZNTS), donde la mayoría de su población es inmigrante, en su mayoría marroquí, con problemas de cohesión social, reúne un potencial educativo y social para que nuestros estudiantes universitarios puedan vincularse y enseñar en participación ciudadana.

La población de este barrio almeriense es un mosaico cultural con identidades plurales y diversas, por lo que desde la educación para la ciudadanía y la enseñanza de las Ciencias Sociales intentamos desarrollar y aplicar este concepto amplio de ciudadanía que 
haga visible la pluralidad, la alteridad y la diversidad presentes en este contexto, que por otra parte son características básicas de la actual democracia (Pagès y Santisteban, 2010).

Desde el planteamiento anterior y como docentes universitarios nos pareció interesante incorporarnos a este Proyecto Socioeducativo Comunitario para la Recuperación de "El Ingenio-El Puche" iniciado en el año 2009, donde la apropiación positiva de los espacios públicos en situaciones complejas de hábitat, como las que se dan en zonas urbanas con problemas de inseguridad y exclusión social, precisa de una amplia intervención y participación social. En las aulas de la universidad trabajamos con nuestro alumnado la importancia del entorno, la realidad próxima y lejana para la experimentación, investigación y aplicación del medio integrado. Y desde la integración se abordaron los dos grandes ejes de la enseñanza de las Ciencias Sociales, el espacio y el tiempo. De esta forma fuimos conectando con nuestro objetivo, unas prácticas profesionalizadoras críticas y de innovación educativa mediante el conocimiento social.

Al tratarse de un proyecto de construcción de Escuela Democrática donde se promueve la intervención social de los habitantes del barrio, fue de gran interés trabajar con el alumnado universitario el sentido y desarrollo de la competencia social y ciudadana en el currículum social.

En la recuperación y apropiación de ese espacio público, el elemento simbólico e identitario era y es un resto patri- monial "La puerta del Ingenio" que nos habla de un pasado que está presente donde podíamos y deseábamos intervenir en su transformación, al tiempo que nos permitía trabajar los conceptos y procedimientos de la temporalidad. Creemos que el aprendizaje del tiempo histórico debe basarse en las relaciones entre pasado, presente y futuro, sobre todo ese futuro posible y deseable tan ausente en la práctica escolar.

Ese espacio al que nos referimos es un solar de grandes dimensiones en su momento descuidado, lleno de basura, llamado Plaza del Ingenio y situado en la zona suroeste del barrio. Su denominación viene de la monumental portada de una antigua fábrica de azúcar que data de 1885. Su función fue cambiando con el paso del tiempo, siendo cárcel durante la II República, la Guerra Civil y el Franquismo; posteriormente se dedicó a la fabricación de productos químicos para finalmente ser abandonada. Las transformaciones en el tiempo de ese espacio nos permitió analizar en el aula conceptos temporales de cambio y continuidad así como iniciar al alumnado en el trabajo de las fuentes, de su uso didáctico, tanto de las fuentes orales, como las documentales, visuales, prensa histórica, así como el uso didáctico de los archivos, tanto municipales como provinciales. Y por supuesto, sin olvidar la perspectiva de género, es decir preguntándonos siempre por las mujeres como sujetos históricos y sociales. En este sentido, la denominación de las calles del barrio con nombres de mujeres nos permitió indagar y visibilizar 
la presencia femenina. Fue un recurso muy motivador para el alumnado de magisterio y para el alumnado de los colegios del barrio.

La Plaza del Ingenio era y es un lugar con unas condiciones óptimas para albergar un espacio común de encuentro y convivencia y en su recuperación se pretendió que participara toda la comunidad del barrio. Esta participación promovió entre la población un sentido de vinculación y pertenencia, favoreció la unión y acercamiento entre sus habitantes y con el resto de la ciudadanía, desapareciendo esa frontera invisible que marca los rasgos distintivos de un contexto de exclusión social.

\section{Ejes vertebradores de nuestro curriculum social crítico}

Pensamos que uno de los retos que presenta el tratamiento de las Ciencias Sociales en las sociedades actuales consiste en superar la construcción de un conocimiento escolar sustentado en la fugacidad y la inmediatez. Vivimos en una sociedad donde no siempre abundan las oportunidades para una reflexión sosegada y crítica del presente. Se corre así el riesgo de familiarizarnos más con un tipo de percepción fragmentaria de la realidad social del presente que con la formación de un pensamiento histórico pausado y más complejo. De esta manera, y dentro del ámbito de la educación formal, los centros educativos no pueden quedar al margen de lo que se vive en la realidad, viviendo de espaldas a los cambios socio-políticos y culturales que se están fraguando en el exterior.

Por todas estas razones, las diferentes áreas de conocimiento escolar de Educación Primaria están obligadas a realizar ese esfuerzo de renovación en la selección de sus contenidos partiendo del currículo establecido. Así, la enseñanza de las Ciencias Sociales que aquí defendemos se sitúa dentro del modelo crítico que focaliza su atención en los problemas sociales relevantes. Estamos de acuerdo con Canals y González (2011, 53) cuando sostienen que este modelo interpreta que el currículo "debe estar centrado en los problemas sociales relevantes para favorecer la formación del pensamiento reflexivo, crítico y creativo, el análisis de los valores y de las prácticas sociales, y el aprendizaje de la toma de decisiones y la resolución de problemas".

Situándonos en el aula de Primaria, ésta puede convertirse en un espacio público tanto para el debate de cierta profundidad como para el estudio de las vivencias de un alumnado sobre la resolución de problemas sociales ya que forma parte de una ciudadanía en ciernes y, aunque legalmente no le sea reconocido el derecho a votar, experimenta asimismo un proceso de enseñanza y aprendizaje de un modelo restringido de ciudadanía tanto en la educación no formal como informal. El aula de conocimiento del medio ha de asumir su función de filtro y de interpretación sosegada de un volumen ingente de información que, a veces, puede ser percibida con rechazo por parte del alum- 
nado. Por tanto, el o la futura docente debería desarrollar la responsabilidad ética y política de convertir su aula en un laboratorio, que estudie el objeto socio cultural e histórico que penetra con fuerza en cualquier espacio público, sobre todo, a través de los medios de comunicación de masas. Su labor, nuestra labor, por tanto, ha de ser la de presentar, ordenar, discernir, estructurar, traducir la información para que sea analizada con rigor desde distintos enfoques y dentro del extenso campo de conocimiento de las Ciencias Sociales.

Como se podrá comprobar más adelante, creemos que el currículo recogido en la Orden del 10 de agosto de 2007, BOJA 30/08/2007 de Primaria en Andalucía propiciaba un escenario de análisis y de racionalidad crítica del presente. Estamos convencidos de la potencialidad de los ámbitos temáticos de las asignaturas de Didáctica de las Ciencias Sociales para fomentar un conocimiento social escolar. Al comprobar que es posible el tratamiento de problemas relevantes en el ámbito escolar de Primaria podemos también desmitificar a las democracias ya consolidadas para que éstas mejoren en su funcionamiento. Nuestra democracia está plenamente asentada en un escenario como el de la Europa occidental. Sin embargo, como síntoma de que la democracia ha de cuidar su salud, hemos de utilizar la educación formal y obligatoria como un proceso necesario para favorecer una emancipación humana y política, no parcial, que se sitúe más allá de la apariencia, a pesar de planteamientos ideológicos conservadores que niegan esta posibilidad en Primaria cuando se adoptan medidas para hacer desaparecer una materia como educación para la ciudadanía. De ahí la importancia que concedemos al desarrollo de la competencia social y ciudadana en la educación obligatoria, ahora social y cívica. Como ya apuntábamos al principio de este trabajo, en la actualidad, vivimos un momento delicado para todo sistema democrático. El edificio de este sistema presenta grietas por donde penetran los riesgos de ciertas patologías escasamente democráticas que se convierten en fantasmas que, como sostiene Espósito (1996), están dispuestos a despertarse.

Nos sumamos a la iniciativa de nuestras compañeras del área de Didáctica y Organización Escolar de la Facultad de Educación de la Universidad de Almería que habían comenzado a colaborar con los centros educativos y las asociaciones del barrio en el curso 2009/10. Una preocupación común fue la necesidad de vincular el conocimiento teórico académico trabajado en las aulas de la universidad y las prácticas educativas. Llevar a la acción este propósito era/es el camino a seguir para renovar el modelo formativo profesionalizador más allá de los cánones tradicionales de la educación formal.

El modelo formativo que hemos compartido se ha enmarcado en la concepción de las escuelas democráticas, entendiendo que éstas posibilitan la participación real de la comunidad educativa (profesorado, alumnado, familias, etc.) en la gestión, organización y desarrollo del currículum. Esto es, formar a nues- 
tros estudiantes universitarios en un modelo democrático, tal como se recogía en la Ley Orgánica de Educación (LOE, 2006), que señala la educación como el medio más adecuado para garantizar el ejercicio de la ciudadanía democrática, responsable, libre y crítica para la constitución de sociedades avanzadas y justas. Al mismo tiempo, adquirir conocimientos, competencias y valores que sustentan la práctica de una ciudadanía democrática, como señalaba la Ley Orgánica de Educación, sólo es posible si se posibilitan espacios reales a todos los integrantes de la comunidad educativa (profesorado, familias, alumnado...) para la participación, el diálogo y la toma de decisiones en lo que afecta a la cotidianidad de los centros educativos, y a los problemas y realidades de las personas.

El currículo de la educación formal en las etapas de educación obligatoria, nacido con la LOE, que estuvo vigente en nuestro país hasta la aplicación definitiva de la LOMCE (BOE, 1 de marzo de 2014) por parte del gobierno del Partido Popular, fue un medio adecuado para ser aprovechado con la intención de cumplir una serie de finalidades educativas, entre ellas la de favorecer la construcción del pensamiento socio-histórico. Concretamente, en la Comunidad Autónoma de Andalucía, el desarrollo curricular de Primaria encarna unas potencialidades en el área de conocimiento del medio relacionada con las Ciencias Sociales que no podemos obviar. Si nos fijamos en el diseño curricular de esas etapas educativas (BOJA, número 171, 30 de agosto 2007), éste no se ha quedado en un plano de mera declaración de intenciones. En esta línea, en Primaria, el área de conocimiento del medio natural, social y cultural se centra en el tratamiento de problemas relevantes que pueden orientar los proyectos educativos de cada centro.

El trabajo voluntario desarrollado por nuestro alumnado en los centros educativos se concretó de forma coordinada con el profesorado de los mismos en unos centros de interés específicos: uno, conocer la historia del barrio desde los años 70 hasta nuestros días; dos, analizar las distintas asociaciones que han estado trabajando durante esos años y trabajan hoy para mejorar la calidad de vida y de la convivencia de sus habitantes y tres, la creación de una conciencia identitaria mediante el elemento patrimonial del barrio. Creemos que esto es muy relevante ya que todo alumnado que finaliza la educación obligatoria ha de desenvolverse socialmente, conocer los rasgos que caracterizan a la sociedad actual; por ello fue esencial propiciar debates en las aulas haciendo patente soluciones posibles a los problemas de convivencia. Por lo tanto, el conocimiento del pasado desde un ángulo más abierto y crítico supone la estimulación de habilidades sociales para que podamos vivir el presente desde una posición más activa y menos pasiva; significa también que se enriquezca la construcción de las identidades personales a la hora de afrontar las situaciones del presente conociendo de antemano las consecuencias del 
pasado reciente; implica, además, una concienciación mayor de los valores del respeto, la escucha, la empatía...Supone, en definitiva, conocer situaciones de injusticia y observar los esfuerzos de distintas asociaciones para favorecer la igualdad de derechos.

\section{La autoevaluación como base reflexiva para la mejora de la formación docente en Didáctica de las Ciencias Sociales}

Hemos concebido la evaluación del trabajo desarrollado por el alumnado de Didáctica de las Ciencias Sociales que ha participado voluntariamente en este Proyecto no como una etapa finalista sino como una vía de inicio para que se interiorice la necesidad de realizar periódicamente autoevaluaciones sobre la práctica docente, resaltando en este sentido la aportación de los diferentes grupos de discusión, entrevistas, cuestionarios y la valoración de las experiencias a través de diarios personales, memorias y diseños curriculares. Estamos convencidos de que estas acciones de autoevaluación propician la construcción de ese espacio de reflexión permanente para la mejora de la profesión docente en Primaria. Al mismo tiempo que contribuye a la evaluación global del programa. Conscientes de los rasgos clave que caracterizan la investigación-acción (Sandín, 2003), hemos centrado más nuestra atención en el proceso para mejorar la práctica educativa. En cada uno de los cursos académicos en los que hemos llevado a cabo esta experiencia, las fases que hemos experimentado han sido cuatro: a) coordinación y negociación con las otras áreas de conocimiento de nuestro ámbito de participación tanto en el Proyecto Socioeducativo como en el Proyecto de Trabajo de Grupo Docente de Innovación para establecer los objetivos a cumplir; b) planteamiento de la propuesta formativa innovadora al alumnado de nuestra asignatura y explicación de las estrategia a seguir; c) fase de aplicación y desarrollo práctico de dicha propuesta en los CEIP del barrio de El Puche; d) recogida de información y análisis de resultados a través de la evaluación.

En esta última fase hemos convertido la evaluación en una puerta abierta para favorecer la mejora de la práctica docente, tanto del formador universitario como del estudiante universitario con la intención de generar una nueva cultura evaluadora amparada en el intercambio de experiencias entre formador universitario, alumnado universitario de Grado y docentes de Primaria en ejercicio. Es evidente que llevar a cabo una evaluación formativa de ese calibre precisaría un tiempo más dilatado para recoger una información rica y variada realizando para ello un análisis longitudinal a lo largo de estos años de experimentación con el fin de establecer un juicio de valor equilibrado sobre la práctica educativa.

Nuestra experiencia nos ha permitido abrir nuevas vías de comunicación entre profesorado y alumnado a la hora de concebir la evaluación desde un punto 
de vista más dinámico y enriquecedor para ambas partes. En este sentido, desde nuestra didáctica específica, mostraremos al final de este apartado varios fragmentos que resumen el contenido de las reflexiones hechas por el alumnado participante en este Programa sobre la vinculación entre teoría y práctica, la hegemonía del modelo tradicional de enseñanza, la vocación docente, la aportación del Aprendizaje Servicio (ApS) y el desarrollo de las competencias-capacidades como maestra/o.

Este trabajo nos ha propiciado un escenario adecuado para la observación y estudio de unas experiencias formativas en un contexto educativo determinado. Creemos que su análisis ha incidido de manera positiva, a pesar de las dificultades, en el reforzamiento de una futura práctica docente adaptada a cualquier escenario educativo, finalidad de cualquier proceso evaluativo. Además, con este enfoque hemos podido disolver una herencia evaluativa tradicional dirigida "casi exclusivamente a medir resultados finales de aprendizaje" (Calatayud, 2007).

Dicho lo anterior, exponemos a continuación los aspectos que fueron evaluados: a) grado de participación, compromiso e implicación personal del alumnado universitario en el Programa; b) su nivel de reflexión crítica, c) su nivel de aprendizaje como futuro/a docente, d) el grado de vinculación desarrollado entre teoría y práctica en el marco de la Didáctica de las Ciencias Sociales, y, d), su volumen de adquisición de competencias y capacidades como docentes de Pri- maria. En este sentido, nos han sido útiles las preguntas que hemos planteado en las encuestas, entrevistas y grupos de discusión para sondear su experiencia formativa en la que se vinculaban universidad, comunidad y escuela.

En este espacio hemos asumido nuestra responsabilidad como docentes formadores teniendo como marco de referencia la Didáctica de las Ciencias Sociales. Todo ello nos ha hecho conscientes del impacto que este conjunto de influencias ha podido dejar en el o la docente futura. Igualmente, a la hora de evaluar a este alumnado universitario, contemplamos positivamente el hecho de que se acogieran voluntariamente a la participación en este Programa Socioeducativo, sin olvidar por ello su madurez a la hora de recibir y mostrar los conocimientos de la materia. Debemos añadir que esta experiencia también se ha convertido en una ocasión favorable para la autoevaluación de nuestra práctica docente como docentes universitarios preocupados por la innovación educativa, teniendo presente, sobre todo, cuáles han de ser las principales finalidades de la Didáctica de las Ciencias Sociales en la formación del profesorado: "Enseñar el oficio de enseñar ciencias sociales (...)” partiendo, entre otros, "de los conocimientos que emergen de la práctica de enseñar contenidos sociales" (Pagès, 2000).

Dentro de las diversas modalidades de la investigación-acción, podemos decir que en este recorrido hemos puesto en práctica tanto la investigación-acción participativa como la colaborativa (San- 
dín, 2003). La participación de un total de 50 estudiantes a lo largo de estos tres cursos académicos, preocupados por fortalecer su formación como maestros y maestras de Primaria, nos ha dado la oportunidad de ajustar nuestro conocimiento sobre las repercusiones positivas de las propuestas formativas innovadoras. Así, si analizamos los datos obtenidos en nuestra investigación, un $20 \%$ del total de participantes en la muestra (10 personas) nos ha manifestado abiertamente que han encontrado barreras en parte del profesorado de los centros que no "creían en el cambio", y eso les ha impedido vivir la innovación educativa en estos contextos. Sin embargo, el $80 \%$ (40 personas) nos ha comunicado que esta experiencia ha sido muy enriquecedora personal y profesionalmente, les ha dado la posibilidad para reflexionar y poner en práctica nuevos aprendizajes para enseñar Ciencias Sociales en Primaria y creer que otra modelo de enseñanza es posible.

Para finalizar este apartado, traemos hasta aquí una muestra resumen de respuestas dadas por el alumnado que consideramos son las más representativas de esta evaluación inicial. Para clarificar y dar sentido a estos testimonios estas son algunas de las preguntas que les planteábamos en las entrevistas y en los grupos de discusión: ¿Has podido vincular teoría y práctica de la asignatura de Didáctica de las Ciencias Sociales al participar en el Programa Socioeducativo de El Puche? ¿Dónde lo has notado? ¿En qué parte has podido comprobar que esto ha sido posible?
¿En qué aspectos no has podido ponerla en práctica? ¿Dónde has encontrado las dificultades para su aplicación? ¿Cómo se podrían resolver?

1. "He podido vincular la teoría y la práctica a la hora de escoger los conceptos (democracia, asociación) y los procedimientos que quería llevar a cabo con "mis alumnos/as", pero sobretodo, me he dado cuenta de lo importante que son las Ciencias Sociales en el currículo ya que nos ayudan a entender la realidad. Sinceramente, pienso que ha faltado tiempo y, en mi caso, dar con un tutor/a que realmente creyese en el cambio y en una posible transformación." (Alumna A.O.)

2. "Sobre todo me ha dado ocasión para encajar piezas de un puzle, como es la organización de los contenidos, que se pueden relacionar con las Ciencias Sociales y cómo aproximarte a ellos en la práctica docente. Es decir, sin darnos cuenta, en muchas ocasiones estamos trabajando contenidos de las Ciencias Sociales pero no somos conscientes de que son objeto de estudio de las Ciencias Sociales y que tienen vías de aproximación definidas como son a través de problemas relevantes o por medio de los conceptos clave. Es cierto, que cuando lo analizas en un contexto real tienes más ocasión de comprender su significado (quizás es una de las ventajas de los que estamos participando en El Puche). Encuentras que entre los pasos que tú puedas planificar y lo que luego pasa en las sesiones puede haber grandes 
diferencias, pero también te das cuenta de que la vida en el aula es así. Y como hemos visto en Ciencias Sociales, la realidad social es muy compleja. La teoría de la asignatura encaja con la necesidad de acercamiento a la realidad de los estudiantes y el entendimiento de su entorno. Sin ellos, la labor docente se distorsiona más aún. Me ha costado poder encajar el tema que acordamos en el proyecto en la clase. Siento que hay muchos pasos para llegar a comprender la problemática que les planteamos que es la de que ellos influyan en su barrio y el barrio les influye a ellos y que una forma de mejorar el barrio es mejorar la convivencia. Que las personas que se unen con un objetivo común consiguen mucho más y que en nuestra sociedad, la participación ciudadana es una acción dormida pero que es un derecho y un deber que hay que saber que existe y entenderlo para poder comprometerse." (Alumna B.P.).

3. "Yo creo y espero que sí que nuestro aprendizaje-servicio nos sirva a las dos partes, al barrio en sí y a los estudiantes. Pienso que ya sólo nuestra presencia en el barrio ya ayuda a esa transformación en el sentido de que sus vecinos ven que hay gente de afuera que se motiva por venir a trabajar con sus hijos e hijas. Yo creo que eso ya les hace ver la realidad de otra forma. Pude oír de ellos y ellas que el barrio es un mal barrio o un sitio que la gente no quiere vivir allí. Esto es algo que resulta muy difícil de hacerles cambiar de idea. Esta idea de barrio marginal la tienen muy asumida en sus vidas. El servicio prestado es mutuo en el sentido de que ellos nos ayudan a nosotros y nosotros no les hacemos sentirse tan alejados de la realidad con nuestra presencia. Es una pena que no se trabaje más en El Puche, y hablo de justicia social. ¿Por qué no hay colegios que lleven a sus alumnos y alumnas a ver un día cómo trabajan en El Puche? No lo entiendo; bueno sí, ahora sí. Después del otro día en clase, ya entiendo lo del "espejismo de la igualdad" o "el techo de cristal". Cualquiera vale para darse cuenta de cuál es la realidad. Sería fácil caer en el pesimismo y la ingenuidad. Pero si perdemos la esperanza que antes mencionaba de la verdadera educación, perdería mi rumbo, pues: ¿no es esa esperanza de todos y todas la que nos hace levantarnos cada día de la cama?". (Alumno R.V.)

\section{Conclusiones}

En la formación universitaria del profesorado y desde la Didáctica de las Ciencias Sociales pretendemos fomentar la educación democrática de la ciudadanía y la práctica del pensamiento social crítico. Tenemos el convencimiento de que la formación de nuestros estudiantes como futuros docentes de Primaria debe prepararlos para el desarrollo de la competencia social y ciudadana.

El resultado de este proceso de investigación-acción nos ha obligado a reflexionar sobre la práctica de la enseñanza de las Ciencias Sociales en 
Primaria y sus finalidades en contextos desfavorecidos vinculándola ésta en todo momento a la metodología del Aprendizaje Servicio (ApS). A pesar de las dificultades organizativas y de funcionamiento, hemos podido comprobar que nuestros fundamentos teóricos $\mathrm{y}$ prácticos a la hora de enseñar a enseñar Ciencias Sociales en el Grado de Educación Primaria son válidos para: en primer lugar, dotar al futuro maestro de un conocimiento práctico amplio conviviendo con maestros y maestras en activo. Hemos ofrecido una posibilidad real -al margen del marco del Practicum-para que el o la estudiante de este Grado contraste, identifique y evalúe el trabajo desempeñado por maestras y maestros en dos centros educativos públicos de Primaria situados en este barrio marginal de la ciudad de Almería. En segundo lugar, el compromiso y la participación de este alumnado universitario en el Proyecto Socioeducativo ha contribuido positivamente a empoderar a la población escolar de un barrio marginal vivenciando así la metodología del Aprendizaje Servicio.

Creemos que hemos contribuido desde la Didáctica de las Ciencias Sociales a hacer posible que nuestro alumnado universitario comprenda que se puede construir una memoria común mediante la relación pasado, presente y futuro. Que la educación patrimonial permite crear una conciencia de ciudadanía intercultural, siendo en este caso la portada de El Ingenio el elemento simbólico de una identidad colectiva para la transformación de un barrio mosaico de culturas, soñando un futuro mejor conociendo el origen histórico de El Puche en los 70 y trabajando en el entorno urbano con diseño de un parque que mejore la vida de sus habitantes. Nuestra estrategia en la formación del profesorado era y es que los estudiantes identifiquen, se cuestionen y reflexionen sobre los problemas concretos que les surgen en la práctica educativa.

A través de este Programa Socioeducativo de participación ciudadana se han abierto las aulas de la Universidad para que no sean espacios fríos de transmisión de conocimientos sino que nuestro alumnado tenga la oportunidad de reconstruirlos a partir de realidades educativas concretas como las mencionadas en los colegios públicos del barrio de El Puche. Es una nueva vía para cambiar el modelo de formación para el alumnado y para nosotras y nosotros como docentes universitarios. Para el alumnado formándose no sólo para una práctica profesional democrática sino en una práctica profesional democrática (García Gómez, 2011) y, para nosotros, teniendo la posibilidad de experimentar otro modelo de formación inicial.

El Programa sigue en marcha, es complejo y puede abordarse desde miradas caleidoscópicas como la educación en valores, la escuela inclusiva, o la que hemos trazado en estas líneas de la participación ciudadana. Pero también es cierto que es un trabajo que se va reconstruyendo, es un proyecto inacabado de investigación-acción. El balance positivo de esta evaluación inicial nos motiva para seguir implicados en el desarrollo 
de proyectos como este donde se vincula universidad, escuela y comunidad.

\section{Referencias bibliográficas}

ARJONA, A. y CHECA, J.C. (2005). Factores que determinan el proceso de exclusión de los barrios periféricos: el caso de El Puche (Almería). Scripta Nova. Revista electrónica de geografía y ciencias sociales, vol. IX, $\mathrm{n}^{\circ} 186$. Recuperado de http://www.ub.es/geocrit/ sn/sn-186.htm [22 de enero de 2014].

BATLLÉ, R. (2010). Aprendizaje-Servicio. Qué, cómo y para qué. Conferencia impartida en las Jornadas "Transformando la escuela con y para la comunidad", 24 de noviembre. Universidad de Almería.

BOYLE-BAISE, M. y GRANT, C. A. (2004). Citizen/community participation in education. Historic change in terms of engagement. En S. ADLER, Critical issues in social studies teacher education (145-146). Greenwich: Conn.

CALATAYUD, M.A. (2007). La evaluación como instrumento de aprendizaje y mejora. Una luz al fondo. En La evaluación como instrumento de aprendizaje. Técnicas y estrategias. Madrid: Ministerio de Educación y Ciencia.

CANALS, R. y GONZÁLEZ, N. (2011). El currículo de Conocimiento del Medio Social y Cultural, y la formación de competencias. En J. PAGÈS y A. SANTISTEBAN (Coords.), Didáctica del Conocimiento del Medio Social y Cultural en la Educación Primaria. Ciencias Sociales para aprender, pensar y actuar (41-62). Madrid: Editorial Síntesis.

CARBONELL, J. (2011). La pedagogía está en la plaza. Cuadernos de Pedagogía, $\mathrm{n}^{\circ} 414,3$.

CRUZ, M. (2012). Adiós, historia, adiós. El abandono del pasado en el mundo actual. Oviedo: Ediciones Nobel.

ESPOSITO, R. (1996). Confines de lo político. Nueve pensamientos sobre político. Madrid: Editorial Trotta.

GARCÍA, T. (2011). Aportaciones ciudadanas desde el aprendizaje servicio. Universidad, escuela y comunidad conectadas. Revista Interuniversitaria de Formación del Profesorado, $\mathrm{n}^{\mathrm{0}}$ 71, 125-141.

GARCÍA, F.F. (2009). Educar para la participación ciudadana. Un reto para la escuela del siglo XXI. Investigación en la Escuela, $\mathrm{n}^{\circ}$ 68, 5-10.

GARCÍA, F.F. y DE ALBA, N. (2007). Educar en la participación como eje de una educación ciudadana. Reflexiones y experiencias. Didáctica Geográfica. $2^{\mathrm{a}}$ época, $\mathrm{n}^{\circ}$ 9, 243-258.

MARTÍNEZ, J.B. (2010). Formación ciudadana y formación en valores. En F. IMBERNON (Coord.), Procesos y contextos educativos: enseñar en las instituciones de educación secundaria. (181-212). Barcelona: Graó.

MORIN, E. (2001). Los siete saberes necesarios de la educación del futuro. Barcelona: Paidós. Recuperado de http://unesdoc.unesco.org/ 
images/0011/001177/117740So.pdf [12 de enero de 2014].

PAGÈS, J. (2000). La didáctica de las ciencias sociales en la formación inicial del profesorado. Íber. Didáctica de las Ciencias Sociales, Geografía e Historia, 24, 33-44.

PAGÈS, J. y SANTISTEBAN, A. (2010). La educación para la ciudadanía y la enseñanza de las ciencias sociales, la geografía y la historia. Íber. Didáctica de las Ciencias Sociales, Geografía e Historia, 64, 8-18.

PUIG, J.M. (coord.) (2009). Aprendizaje y servicio. Educación y compromiso cívico. Barcelona: Graó.
RAMONEDA, J. La democracia en peligro, El País, 16 de enero de 2012.

SÁNCHEZ, M ${ }^{\mathrm{a}}$. S. y GARCÍA, T. (Coords.) (2010). Vinculando TeoríaPráctica: El aprendizaje servicio para la formación y la transformación. En AA.VV. Programa Socioeducativo de Carácter Comunitario para la Recuperación de "El Ingenio - El Puche".

SANDÍN, M.P.(2003). Investigación cualitativa en educación. Fundamentos y tradiciones. Madrid: McGraw Hill.

TEDESCO, J.C. (2000). Educar en la sociedad de conocimiento. Buenos Aires: Fondo de Cultura Económica. 
